for changes in antibiotic use. Results: The data included 7,094 patients in the preintervention group and 6,661 patients in the postintervention group. The BPA fired 1,478 times. The prescribing rate of piperacillin-tazobactam 1 year prior to the BPA was $32.34 \mathrm{DDD}$ and decreased every month both before $(-1.22$ DDD) and after ( -0.27 DDD) the BPA initiation, with no significant difference in prescribing trends $(P=.10)$. Meropenem prescribing in the BPA era increased each month compared to the pre-BPA period (1.16 DDD; $P=0.02)$, whereas cefazolin use $(P=.93)$ and ceftriaxone $(P=.09)$ use did not significantly change. Conclusions: The data show that piperacillin-tazobactam utilization at our institution is decreasing. Considering that this trend started prior to the launch of the BPA and that rate of decline remained unchanged post-BPA, we conclude that the BPA did not further impact our piperacillin-tazobactam consumption. It is possible that other factors influencing prescribing account for the observed decline, including an institution-wide educational campaign regarding the appropriate use of broad-spectrum antibiotics that was initiated in the months prior to the BPA. The reason for the significant rise in meropenem post-BPA is unclear. This may be unrelated to the BPA; however, it requires further investigation.

1. Core elements of hospital antibiotic stewardship programs. Centers for Disease Control and Prevention website. https:// www.cdc.gov/antibioticuse/healthcare/implementation/coreelements.html. Updated July 19, 2019. Accessed October 6, 2019. Funding: None

\section{Disclosures: None}

Doi:10.1017/ice.2020.1057

\section{Presentation Type:}

Poster Presentation

The Impact of Changing to an Algorithm-Based Clostridioides difficile Test on the Decision to Treat Clostridioides difficile

Lana Dbeibo, Indiana University School of Medicine; Joy Williams, Indiana University School of Medicine; Josh Sadowski, Indiana University Health; William Fadel, Indiana University; Vera Winn, Indiana University Health; Cole Beeler; Kristen Kelley; Douglas Houston Webb, Indiana University Health; Areeba Kara, Indiana University School of Medicine

Background: Polymerase chain reaction (PCR) testing for the diagnosis of Clostridioides difficile infection (CDI) detects the presence of the organism; a positive result therefore cannot differentiate between colonization and the pathogenic presence of the bacterium. This may result in overdiagnosis, overtreatment, and risking disruption of microbial flora, which may perpetuate the CDI cycle. Algorithm-based testing offers an advantage over PCR testing as it detects toxin, which allows differentiation between colonization and infection. Although previous studies have demonstrated the clinical utility of this testing algorithm in differentiating infection from colonization, it is unknown whether the test changes CDI treatment decisions. Our facility switched from PCR to an algorithm-based testing method for CDI in June 2018. Objective: In this study, we evaluated whether clinicians' decisions to treat patients are impacted by a test result that implies colonization (GDH+/Tox-/PCR + test), and we examined the impact of this decision on patient outcomes. Methods: This is a retrospective cohort study of inpatients with a positive $C$. diff test between June 2017 and June 2019. The primary outcome was the proportion of patients treated for CDI. We compared this outcome in 3 groups of patients: those with a positive PCR test (June 2017June 2018), those who had a GDH+/Tox-/PCR + or a GDH+/ Tox + test result (June 2018-June 2019). Secondary outcomes included toxic megacolon, critical care admission, and mortality in patients with $\mathrm{GDH}+/ \mathrm{Tox}-/ \mathrm{PCR}+$ who were treated versus those who were untreated. Results: Of patients with a positive PCR test, $86 \%$ were treated with CDI-specific antibiotics, whereas $70.4 \%$ with GDH $+/$ Tox + and $29.25 \%$ with GDH $+/$ Tox $-/ P C R+$ result were treated $(P<.0001)$. Mortality was not different between patients with GDH+/Tox-/PCR + who were treated versus those who were untreated $(2.7 \%$ vs $3.4 \% ; P=.12)$, neither was critical care admission within 2 or 7 days of test result (2\% vs $1.4 \%$; $P=.15)$ and $(4.1 \%$ vs $5.4 \%, P=.39)$, respectively. There were no cases of toxic megacolon during the study period. Conclusions: The change to an algorithm-based C. difficile testing method had a significant impact on the clinicians' decisions to treat patients with a positive test, as most patients with a GDH+/Tox-/ $\mathrm{PCR}+$ result did not receive treatment. These patients did not suffer more adverse outcomes compared to those who were treated, which has implications for testing practices. It remains to be explored whether clinicians are using clinical criteria to decide whether or not to treat patients with a positive algorithm-based test, as opposed to the more reflexive treatment of patients with a positive PCR test.

Funding: None

Disclosures: None

Doi:10.1017/ice.2020.1058

Presentation Type:

Poster Presentation

The Impact of Intervention-Related Risk Factors on the Risk of Ventilator-Associated Pneumonia Is High in a Neurosurgical Intensive Care Unit

Ksenia Ershova, Keck School of Medicine at USC; Oleg Khomenko, Skolkovo Institute of Science and Technology; Olga Ershova, Burdenko Neurosurgery Institute; Ivan Savin, Burdenko Neurosurgery Institute; Natalia Kurdumova, Burdenko Neurosurgery Institute; Gleb Danilov, Burdenko Neurosurgery Institute; Michael Shifrin, Burdenko Neurosurgery Institute

Background: Ventilator-associated pneumonia (VAP) represents the highest burden among all healthcare-associated infections (HAIs), with a particularly high rate in patients in neurosurgical ICUs. Numerous VAP risk factors have been identified to provide a basis for preventive measures. However, the impact of individual factors on the risk of VAP is unclear. The goal of this study was to evaluate the dynamics of various VAP risk factors given the continuously declining prevalence of VAP in our neurosurgical ICU. Methods: This prospective cohort unit-based study included neurosurgical patients who stayed in the ICU $>48$ consecutive hours in 2011 through 2018. The infection prevention and control (IPC) program was implemented in 2010 and underwent changes to adopt best practices over time. We used a 2008 CDC definition for VAP. The dynamics of VAP risk factors was considered a time series and was checked for stationarity using theAugmented Dickey-Fuller test (ADF) test. The data were censored when a risk factor was present during and after VAP episodes. Results: In total, 2,957 ICU patients were included in the study, 476 of whom had VAP. Average annual prevalence of VAP decreased from 15.8 per 100 ICU 